\title{
Polysaccharides Produced by Cystobacter, Archangium, Sorangium and Stigmatella Species
}

\author{
By I. W. SUTHERLAND \\ Department of Microbiology, University of Edinburgh, West Mains Road, \\ Edinburgh $E H 93 J G$
}

(Received 16 August 1978)

\begin{abstract}
Exopolysaccharides and lipopolysaccharides were prepared from a number of myxobacterial strains representing the more complex types. The exopolysaccharides were isolated from fruiting bodies and from liquid and solid cultures. The polysaccharides secreted by the bacillary forms in solid or liquid media closely resembled the material obtained from fruiting bodies, the monosaccharides present being in the same approximate molar ratios. Many of the sugars present in the exopolysaccharides were also detected in the lipopolysaccharides, suggesting an economic use of sugar nucleotide synthetic systems. Several, but not all, lipopolysaccharides contained material resembling 3-O-methylxylose in its chromatographic mobility. In addition, a faster-moving spot, as yet unidentified, was noted in some hydrolysates. The commonest monosaccharide components of the lipopolysaccharide were rhamnose, mannose, glucose and galactose. Small quantities of amino sugars, particularly glucosamine and galactosamine, were also detected.
\end{abstract}

\section{INTRODUCTION}

The Myxobacterales differ from other prokaryotic species in their ability to undergo complex developmental cycles. These involve the conversion of the long thin Gramnegative rods to myxospores, resting cells which differ in many respects from the vegetative bacteria. In nature and under suitable laboratory conditions, fruiting bodies are formed. As demonstrated in the elegant studies of Reichenbach (cited in Gerth \& Reichenbach, 1978), the process of fructification arises through cooperative morphogenesis. Large numbers of vegetative bacteria converge to form aggregates which differentiate into the fruiting bodies of varying complexity (Reichenbach \& Dworkin, 1969; Brockman \& Todd, 1974; Grilione \& Pangborn, 1975).

The vegetative bacteria are associated with large amounts of extracellular slime and, in the fruiting body, slime is also present surrounding the myxospores. An earlier study on several strains of Myxococcus species demonstrated the gross chemical similarity between the polysaccharides from vegetative bacteria and those from fruiting bodies in these strains (Sutherland \& Thomson, 1975). Much more marked changes occur in the cell walls during morphogenesis. The appearance of D-glucose, $N$-acetyl-D-galactosamine and glycine in the walls of Myxococcus xanthus cysts was studied by White (1975). Subsequently, the appearance of D-glucose and the amino sugar was confirmed in the myxospores from a range of strains and the glucose was shown to be a component of a 1,3- $\alpha$-linked polymer located on the surface of the resting cells (Sutherland \& Mackenzie, 1977). The work reported here was aimed at determining the nature of the lipopolysaccharides and polysaccharides from myxobacterial species forming complex fruiting bodies.

0022-1287/79/0000-8323 \$02.00 (C) 1979 SGM 


\section{METHODS}

Bacteria. Two strains, Cystobacter velatus Plv9 and Stigmatella aurantiaca Sga10, were kindly provided by Dr Hans Reichenbach, Gesellschaft für Biotechnologische Forschung, Braunschweig-Stöckheim, W. Germany. Other strains were isolated from local soil and from the rotting bark of trees. All strains grew well on Casitone agar (C10 medium of Reichenbach \& Dworkin, 1969) and in Casitone broth in shake flasks at $30^{\circ} \mathrm{C}$, although yields from some strains were low in the liquid medium. With one exception, Sorangium strain C, the bacteria grew in liquid medium as clumps which increased in size with the length of incubation. Fruiting bodies were isolated from cultures grown for 7 to $14 \mathrm{~d}$ on either bacterial cell agar or yeast cell agar (Reichenbach \& Dworkin, 1969).

Polysaccharide production and isolation. Lipopolysaccharides were prepared, using the phenol procedure, from bacteria that had been grown in liquid Casitone medium, then lyophilized and extracted with chloroform/methanol (2:1, v/v) (Sutherland \& Smith, 1973). The product was purified by ultracentrifugation at $100000 \mathrm{~g}$ for $4 \mathrm{~h}$. Lipid A was removed by hydrolysis in $1 \%(\mathrm{v} / \mathrm{v})$ acetic acid for $1 \mathrm{~h}$ at $100{ }^{\circ} \mathrm{C}$. The hydrolysate was dialysed against distilled water, lipid was removed by low-speed centrifugation and the clear, colourless supernatant fluid was lyophilized.

Exopolysaccharides from bacteria grown on solid media were obtained after 5 to $6 \mathrm{~d}$ incubation at $30^{\circ} \mathrm{C}$. The humidity of the culture medium was maintained by incubating all plates in sealed polythene bags. Large $(14 \mathrm{~cm}$ diam.) Petri dishes with a $1 \mathrm{~cm}$ depth of medium were normally used. The bacteria and associated exopolysaccharide were carefully removed from the surface of the medium and suspended in a small volume of saline containing $0.1 \%(\mathrm{v} / \mathrm{v})$ formaldehyde. Polysaccharide was removed from the bacteria by stirring in a high-speed vortex mixer (MSE). The bacteria were sedimented by centrifugation at $10000 \mathrm{~g}$ for $20 \mathrm{~min}$ and the supernatant fluid was added to 2 vol. acetone at $-20^{\circ} \mathrm{C}$. The precipitate was washed thoroughly with cold acetone and dried under vacuum. The product was purified by phenol treatment followed by dialysis, reprecipitation with acetone and drying under vacuum. Polysaccharides were prepared from liquid culture and from fruiting bodies as previously described (Sutherland \& Thomson, 1975).

Analytical methods. All micromethods were sensitive to 5 to $10 \mu \mathrm{g}$ monosaccharide, thus permitting the study of the relatively small amounts of material available from the fruiting bodies. After hydrolysis in $0.5 \mathrm{M}^{-} \mathrm{H}_{2} \mathrm{SO}_{4}\left(100^{\circ} \mathrm{C}\right.$ for $\left.6 \mathrm{~h}\right)$ and neutralization with saturated $\mathrm{Ba}(\mathrm{OH})_{2}$ solution, hydrolysates were separated into neutral and basic fractions by preparative paper electrophoresis on Whatman $3 \mathrm{MM}$ paper in pyridinium acetate buffer (pyridine/acetic acid/water; $10: 4: 86$, by vol.) pH $5 \cdot 3$. No acid material was detected.

The purpose of the electrophoretic separation was to enable the galactose oxidase reagent to be used for the estimation of both galactose and galactosamine. D-Glucose in the hydrolysates was estimated using the glucose oxidase reagent, and D-mannose was estimated by the hexokinase procedure after the destruction of glucose and galactose with the respective oxidases. Other sugars and total hexose were determined on unhydrolysed material by the procedures used in an earlier study (Sutherland \& Smith, 1973) or by the phenol-sulphuric acid method of Dubois et al. (1956).

Periodate oxidation was performed with $24 \mathrm{mM}$-sodium metaperiodate in $0 \cdot 1 \mathrm{M}$-acetate buffer (pH 5.0) for $5 \mathrm{~d}$ in the dark at room temperature. Oxidation was terminated by adding ethylene glycol, and the oxidized polymer was recovered after dialysis and lyophilization.

The monosaccharides in acid hydrolysates of polysaccharides and lipopolysaccharides were examined by descending paper chromatography in butan-1-ol/pyridine/water (6:4:3, by vol.) (solvent A), ethyl acetate/acetic acid/pyridine/water (5:1:5:3, by vol.) (solvent B) or ethyl acetate/acetic acid/formic acid/water (18:3:1:4, by vol.) (solvent C). Paper electrophoresis was done using Locarte (London) equipment with an $80 \times 20 \mathrm{~cm}$ cooled plate area at a current of 90 to $100 \mathrm{~mA}$. Sugar spots on chromatograms or electropherograms were revealed with alkaline silver nitrate reagent (dipping in $1 \mathrm{ml}$ saturated aqueous $\mathrm{AgNO}_{3}$ in $200 \mathrm{ml}$ acetone, followed by dipping in $10 \mathrm{~g} \mathrm{NaOH}$ dissolved in $7.5 \mathrm{ml}$ water and made up to $500 \mathrm{ml}$ with methanol) or by spraying with a saturated aqueous solution of aniline oxalate and heating at $100^{\circ} \mathrm{C}$ for $15 \mathrm{~min}$.

\section{RESULTS AND DISCUSSION}

\section{Polysaccharides}

The yields of polysaccharides and myxospores from fruiting bodies of the strains examined are shown in Table 1. Also included for comparison are values for two strains of Myxococcus which were examined earlier (Sutherland \& Thomson, 1975). The results presented are the mean of four to five preparations and were surprisingly consistent. As 
Table 1. Yields of polysaccharides and myxospores from fruiting bodies

$\quad$ Culture
Archangium $\mathrm{sp} . \mathrm{W} 3$
Archangium $\mathrm{sp}$. P
Cystobacter velatus Plv9
Myxococcus sp. E
Myxococcus xanthus FB
Sorangium sp. A
Stigmatella aurantiaca Sga10

Yield ( $\%$ dry wt of fruiting bodies)

$\begin{array}{cc}\text { Myxospores } & \text { Polysaccharide } \\ 91.3 & 8.7 \\ 84.9 & 15.0 \\ 85.9 & 14.0 \\ 85.9 & 14.0 \\ 84.5 & 15.4 \\ 87.4 & 12.5 \\ 88.7 & 11.2\end{array}$

Table 2. Yields of exopolysaccharide and lipopolysaccharide from vegetative cells

Strains were grown on solid or liquid Casitone medium; cells were recovered by centrifugation and lyophilized. Polysaccharide was recovered by acetone precipitation, purified by phenol treatment and lyophilized. Lipopolysaccharides were extracted with phenol from bacteria after preliminary extraction of lipids with chloroform/methanol.

\begin{tabular}{lcc}
\multicolumn{1}{c}{ Culture } & \multicolumn{2}{c}{ Yield (\% dry wt of cells) } \\
\cline { 2 - 3 } Archangium sp. W3 & $1 \cdot 03$ & $24 \cdot 3$ \\
Archangium sp. P & $1 \cdot 23$ & $28 \cdot 9$ \\
Cystobacter velatus Plv9 & $1 \cdot 32$ & $28 \cdot 5$ \\
Myxococcus sp. E & $1 \cdot 47$ & $8 \cdot 1$ \\
Myxococcus xanthus FB & $0 \cdot 70$ & $10 \cdot 8$ \\
Sorangium sp. A & $1 \cdot 93$ & $22 \cdot 7$ \\
Sorangium sp. C & $1 \cdot 27$ & $20 \cdot 1$ \\
Stigmatella aurantiaca Sga10 & $1 \cdot 31$ & $27 \cdot 4$
\end{tabular}

only a small yield of Sorangium C fruiting bodies was obtained, the values were not considered accurate enough for inclusion, but they appeared to show the same ratio of myxospores to polysaccharide as the other strains examined. The polysaccharide also contained the same monosaccharides as did the preparations from the same strain grown in solid and liquid medium. The yields of polysaccharide obtained from vegetative cells are given in Table 2. Again, values for the two Myxococcus strains are included for comparison. Clearly, more polysaccharide was produced by the strains in the present study than by the Myxococcus strains. The reason for this is not certain, but may perhaps be related to the relatively slow growth and non-dispersed nature of the cultures in liquid medium.

The composition of the polysaccharides was first determined by chromatography of hydrolysates (solvents A and B). Analysis of the polysaccharides (Table 3) indicated that rhamnose, D-mannose, D-galactose and D-glucose are the commonest component sugars, although both the Sorangium preparations lacked rhamnose. No major differences were found between the different polysaccharide preparations from the same strain, indicating that, as for the Myxococcus xanthus and other strains of Myxococcus examined earlier (Sutherland \& Thomson, 1975), material from the fruiting bodies is probably the same as that synthesized by vegetative bacilli. This perhaps indicates that the polysaccharide found within the fruiting bodies is all formed by the bacilli before they undergo conversion to myxospores. This is to be expected if the myxospores, as resting forms, have a reduced biosynthetic capability. Despite the number of sugars common to the polysaccharides produced by the different cultures, some differences are apparent, particularly in the results for the oxidized polymers. Many of the monosaccharides were resistant to oxidation, indicating either the presence of 1,3 linkages or a high degree of branching with substitution at the 2 and 4 positions. This might account for the high viscosity noted for some of the polysaccharides in solution, especially that from Stigmatella aurantiaca. It was not clear whether the small amounts of amino sugars detected in some preparations were genuine 
Table 3. Exopolysaccharide composition

\begin{tabular}{|c|c|c|c|c|c|}
\hline \multirow[b]{2}{*}{ Material } & \multicolumn{5}{|c|}{ Composition ( $\%$ of total carbohydrate $\left.{ }^{*}\right)$} \\
\hline & Rha & Man & Glc & Gal & $\mathrm{HexN} \dagger$ \\
\hline \multicolumn{6}{|c|}{ Cystobacter velatus Plv9 } \\
\hline Fruiting bodies & $32 \cdot 6$ & $25 \cdot 3$ & $27 \cdot 8$ & $1 \cdot 1$ & $3 \cdot 2(\mathrm{GalN})$ \\
\hline Solid medium & $37 \cdot 2$ & $26 \cdot 9$ & $26 \cdot 7$ & 0 & $4 \cdot 7$ (GalN) \\
\hline Liquid medium & 36.9 & $23 \cdot 5$ & $25 \cdot 0$ & $0 \cdot 9$ & $3 \cdot 6$ (GalN) \\
\hline Oxidized polymer & $3 \cdot 4$ & $40 \cdot 8$ & $51 \cdot 0$ & 0 & 0 \\
\hline \multicolumn{6}{|l|}{ Sorangium A } \\
\hline Solid medium & - & $11 \cdot 8$ & $28 \cdot 4$ & $50 \cdot 8$ & 0 \\
\hline Liquid medium & - & $10 \cdot 3$ & $25 \cdot 1$ & $58 \cdot 6$ & 0 \\
\hline Oxidized polymer & - & $30 \cdot 7$ & 0 & $43 \cdot 2$ & 0 \\
\hline \multicolumn{6}{|l|}{ Sorangium $\mathrm{C}$} \\
\hline Liquid medium & 0 & $21 \cdot 9$ & $2 \cdot 7$ & $22 \cdot 8$ & $22 \cdot 7(\mathrm{GlcN})$ \\
\hline Oxidized polymer & 0 & 1.9 & $2 \cdot 9$ & $32 \cdot 8$ & 36.9 (GlcN) \\
\hline \multicolumn{6}{|c|}{ Stigmatella aurantiaca Sga10 } \\
\hline Solid medium & $8 \cdot 6$ & - & $51 \cdot 2$ & $31 \cdot 2$ & $6 \cdot 0$ \\
\hline Liquid medium & $9 \cdot 7$ & - & $48 \cdot 5$ & $26 \cdot 4$ & $6 \cdot 1$ \\
\hline Oxidized polymer & 0 & - & $27 \cdot 6$ & $56 \cdot 4$ & 0 \\
\hline \multicolumn{6}{|l|}{ Archangium W3 } \\
\hline Fruiting bodies & $20 \cdot 6$ & $26 \cdot 5$ & $27 \cdot 7$ & $8 \cdot 8$ & $4 \cdot 5(\mathrm{GlcN})$ \\
\hline Solid medium & $21 \cdot 3$ & $29 \cdot 1$ & $28 \cdot 0$ & $7 \cdot 3$ & $3 \cdot 4(\mathrm{GlcN})$ \\
\hline Liquid medium & $19 \cdot 7$ & $26 \cdot 2$ & $29 \cdot 3$ & $10 \cdot 5$ & $4 \cdot 7(\mathrm{GlcN})$ \\
\hline Oxidized polymer & 0 & $24 \cdot \overline{7}$ & $45 \cdot 3$ & $13 \cdot 1$ & 0 \\
\hline \multicolumn{6}{|l|}{ Archangium $\mathbf{P}$} \\
\hline Fruiting bodies & $5 \cdot 7$ & $30 \cdot 4$ & $36 \cdot 5$ & $19 \cdot 7$ & - \\
\hline Solid medium & $1 \cdot 3$ & $32 \cdot 6$ & $33 \cdot 9$ & $18 \cdot 5$ & - \\
\hline Liquid medium & $2 \cdot 3$ & $28 \cdot 5$ & $37 \cdot 1$ & $19 \cdot 3$ & - \\
\hline
\end{tabular}

components of the extracellular polysaccharides or were derived from contaminating lipopolysaccharide. One interesting feature of all the polysaccharides examined was the total absence of uronic acids which are commonly found in exopolysaccharides from other bacterial genera.

\section{Lipopolysaccharides}

In yield and general composition, the lipopolysaccharides and the lipid-free polysaccharides derived from them differed little from those described for other bacterial genera (Tables 2 and 4). One general feature was the absence of heptose, although 2keto-3-deoxyoctonic acid was present. A number of other bacterial groups also lack heptose and thus differ from the well-characterized lipopolysaccharides of the Enterobacteriaceae (Wilkinson, 1977). Of particular interest was the appearance on many of the chromatograms of a spot staining reddish-pink with aniline oxalate and having relative mobilities of $R_{\mathrm{Rha}}$ 1.12 and $R_{\text {Glc }} 1.96$ in solvent $A$ and $R_{\mathrm{Rha}} 1.27$ and $R_{\text {Glc }} 2.31$ in solvent C. This was most probably 3-O-methylxylose which was shown by Weckesser et al. (1971) to be a component of LPS from Myxococcus fulvus. This monosaccharide was clearly absent from some of the preparations so is not a constituent of all myxobacterial lipopolysaccharides. Although it was not quantified, the intensity of the spot attributed to 3-O-methylxylose indicated that the actual content varied considerably in those polymers which contained it. In this respect, it may resemble other $O$-methyl sugars found in photosynthetic bacteria and in cyanobacteria. Tharanathan et al. (1978) indicated that in some of these polymers only trace amounts of such sugars were present - less than $1 \%$ of polymer dry weight - whereas in other strains larger amounts were present. They also suggested that the $O$-methyl sugars 
Table 4. Lipopolysaccharide composition

\begin{tabular}{|c|c|c|c|c|c|c|c|c|c|}
\hline & & & $\mathrm{mp}$ & $o n^{*}$ & tote & arboh & rate) & & \\
\hline Material & Rha & $\mathrm{Xyl}$ & Man & Glc & Gal & GlcN & GalN & MeXyl & ' $Q$ ' \\
\hline Cystobacter velatus & & & & & & & & & \\
\hline LPS & $25 \cdot 4$ & $3 \cdot 4$ & $14 \cdot 6$ & $9 \cdot 6$ & $1 \cdot 1$ & $12 \cdot 6$ & 0 & + & - \\
\hline Oxidized LPS & - & - & - & - & - & - & - & - & - \\
\hline Polysaccharide $\dagger$ & $37 \cdot 1$ & $8 \cdot 9$ & $16 \cdot 8$ & $12 \cdot 4$ & $2 \cdot 3$ & $1 \cdot 1$ & 0 & + & - \\
\hline Sorangium A & & & & & & & & & \\
\hline LPS & - & $1 \cdot 5$ & $8 \cdot 8$ & $9 \cdot 9$ & $38 \cdot 0$ & $9 \cdot 4$ & $3 \cdot 7$ & $\operatorname{tr}$ & - \\
\hline Oxidized LPS & - & 0 & $28 \cdot 6$ & $23 \cdot 7$ & $26 \cdot 9$ & 0 & 0 & - & - \\
\hline Polysaccharide $\dagger$ & - & 0 & $11 \cdot 7$ & $12 \cdot 8$ & $54 \cdot 3$ & 0 & 0 & $\operatorname{tr}$ & - \\
\hline Sorangium $\mathrm{C}$ & & & & & & & & & \\
\hline LPS & - & $11 \cdot 8$ & $33 \cdot 5$ & $10 \cdot 0$ & $12 \cdot 4$ & $14 \cdot 6$ & 0 & + & - \\
\hline Stigmatella auranti & & & & & & & & & \\
\hline LPS & $20 \cdot 9$ & $9 \cdot 7$ & $8 \cdot 1$ & $22 \cdot 2$ & $19 \cdot 8$ & $9 \cdot 8$ & $4 \cdot 8$ & - & + \\
\hline Oxidized LPS & $11 \cdot 1$ & $7 \cdot 4$ & 0 & $18 \cdot 4$ & $36 \cdot 3$ & 0 & 0 & - & - \\
\hline Polysaccharide $\dagger$ & $22 \cdot 6$ & $8 \cdot 8$ & $20 \cdot 5$ & $14 \cdot 0$ & $14 \cdot 7$ & $4 \cdot 9$ & $5 \cdot 1$ & - & + \\
\hline Archangium W3 & & & & & & & & & \\
\hline LPS & $19 \cdot 9$ & $7 \cdot 2$ & $21 \cdot 5$ & $0 \cdot 3$ & $16 \cdot 9$ & $4 \cdot 5$ & $7 \cdot 7$ & + & + \\
\hline Oxidized LPS & $6 \cdot 9$ & $17 \cdot 2$ & $27 \cdot 3$ & $2 \cdot 2$ & $7 \cdot 2$ & 0 & $10 \cdot 1$ & - & - \\
\hline Polysaccharide $†$ & $20 \cdot 4$ & $9 \cdot 3$ & $20 \cdot 7$ & $2 \cdot 8$ & $20 \cdot 8$ & 0 & $8 \cdot 7$ & + & + \\
\hline Archangium $\mathrm{P}$ & & & & & & & & & \\
\hline LPS & $10 \cdot 6$ & $5 \cdot 4$ & $24 \cdot 9$ & $18 \cdot 6$ & $1 \cdot 1$ & $12 \cdot 2$ & $6 \cdot 5$ & - & - \\
\hline
\end{tabular}

-, Not detected chromatographically; tr, trace.

* All LPS preparations contained 2-keto-3-deoxyoctonic acid, detected by the thiobarbituric acid assay; small quantities of unidentified components were present in some preparations, but heptose was absent.

$\dagger$ Lipid-free polysaccharide from $1 \%(\mathrm{v} / \mathrm{v})$ acetic acid hydrolysis.

+ MeXyl, 3-O-Methylxylose.

were present as reducing or non-reducing terminal sugars. The 3-O-methylxylose in the strains examined here was always destroyed by periodate oxidation, so it too may occupy a terminal position in the polysaccharide chain. Of the three strains listed in Table 4 as containing this sugar, Cystobacter velatus, Sorangium C and Archangium W3, probably all contained 4 to $5 \%$ of the sugar while Sorangium A LPS only contained trace amounts.

The discovery of a second fast-moving sugar (designated Q in Table 4) was also of interest. In solvent A its relative mobilities were $R_{\mathrm{Glc}} 2.18$ and $R_{\mathrm{Rha}} 1.24$ and in solvent C, $R_{\mathrm{Rha}} 1.43$ and $R_{\text {Glc }} 2.59$. It stained weakly with alkaline silver nitrate but rather better with aniline oxalate to give a yellowish colour very similar to that obtained with rhamnose and fucose. Tests on material eluted from a preparative chromatogram in solvent A showed that it did not react in either the ferric chloride assay for pentoses or the cysteine-sulphuric acid assay for 5-methylpentoses. The spot did not fluoresce with the aniline oxalate reagent under ultraviolet light and this, together with the $R_{\text {Gle }}$ value, indicates that the sugar is not 3-O-methyl-D-mannose, a fast-moving sugar found in Rhodopseudomonas viridis (Weckesser et al., 1974). Although only found in two of the six strains examined, this new sugar appeared in one LPS, that of Stigmatella aurantiaca, as the sole sugar with faster chromatographic mobility than rhamnose, but in the LPS of Archangium W3 it was present in about the same amount as 3-O-methylxylose. The presence of these two sugars is thus not mutually exclusive. Xylose and mannose were present in all the preparations tested, while rhamnose, glucose, galactose and amino sugars were also frequently found. Periodate oxidation showed differences from the results noted for the exopolysaccharide. Thus, although many sugars are common to LPS and exopolysaccharide, the extracellular polymers are not identical with the 'O-antigen' components of the LPS. There is, nevertheless, considerable overlap in the biosynthetic pathways required for the two types of polymer 
and this may be of advantage to these bacteria when they form aggregates and then fructify under conditions of nutrient deprivation.

The technical assistance of Miss L. Cuthbertson is gratefully acknowledged.

\section{REFERENCES}

Brockman, E. R. \& TodD, R. L. (1974). Fruiting myxobacteria as viewed with a scanning electron microscope. International Journal of Systematic Bacteriology 24, 118-124.

Dubois, M., Gilles, K. A., Hamilton, J. K., Rebers, P. A. \& SMITH, F. (1956). Colorimetric method for determination of sugars and related substances. Analytical Chemistry 28, 350-356.

GERTH, K. \& REICHENBACH, H. (1978). Induction of myxospore formation in Stigmatella aurantiaca (Myxobacterales). I. General characterization of the system. Archives of Microbiology 117, 173182.

Grilione, P. L. \& PANGBoRN, J. (1975). Scanning electron microscopy of fruiting body formation by myxobacteria. Journal of Bacteriology 124, 1558-1565.

ReichenbaCH, H. \& Dworkin, M. (1969). Studies on Stigmatella aurantiaca (Myxobacterales). Journal of General Microbiology 58, 3-14.

Sutherland, I. W. \& MACKenzIE, C. L (1977). Glucan common to the microcyst walls of cystforming bacteria. Journal of Bacteriology 129, 599-605.

Sutherland, I. W. \& Smith, M. L. (1973). The lipopolysaccharides of fruiting and non-fruiting myxobacteria. Journal of General Microbiology 74, 259-266.

Sutherland, I. W. \& Thomson, S. (1975). Com- parison of polysaccharides produced by Myxococcus strains. Journal of General Microbiology 89, 124-132.

Tharanathan, R. N., Mayer, H. \& Weckesser, J. (1978). Location of $O$-methyl sugars in antigenic (lipo-)polysaccharides of photosynthetic bacteria and cyanobacteria. Biochemical Journal 171, 403408.

Weckesser, J., Rosenfelder, G., MAYer, H. \& LÜDERITZ, O. (1971). The identification of 3-Omethyl-D-xylose and 3-O-methyl-L-xylose as constituents of the lipopolysaccharides of Myxococcus fulvus and Rhodopseudomonas viridis respectively. European Journal of Biochemistry 24, 112-115.

Weckesser, J., Drews, G., Roppel, J., Mayer, H. \& Fromme, I. (1974). The lipopolysaccharides (O-antigens) of Rhodopseudomonas viridis. Archives of Microbiology 101, 233-245.

WHITE, D. (1975). Myxospores of Myxococcus xanthus. In Spores VI, pp. 44-51. Edited by P. Gerhardt, R. N. Costilow \& H. L. Sadoff. Washington: American Society for Microbiology.

Wilkinson, S. G. (1977). Composition and structure of bacterial lipopolysaccharides. In Surface Carbohydrates of the Prokaryotic Cell, pp. 97175. Edited by I. W. Sutherland. London: Academic Press. 